\title{
Macrocerebellum in Achondroplasia: A Further CNS Manifestation of FGFR3 Mutations?
}

\author{
(D) H.M. Pascoe, (D).Y.-M. Yang, (D). Chen, (D)A.M. Fink, and (D). Kumbla
}

\begin{abstract}
SUMMARY: Achondroplasia is the result of a mutation in the fibroblast growth factor receptor 3 gene (FGFR3). Appearances suggestive of macrocerebellum have not been described in this patient group. We retrospectively reviewed MR imaging studies of the brain in 23 children with achondroplasia. A constellation of imaging findings that are recognized in macrocerebellum was observed, including cerebellar hemisphere enlargement (inferior and superior extension, wrapping around the brainstem); an effaced retroand infravermian cerebellar subarachnoid CSF space; a shortened midbrain; distortion of the tectal plate; and mass effect on the brainstem. All MR imaging studies exhibited some of these findings. Quantitative analysis confirmed an increased cerebellar volume compared with age- and sex-matched controls. We hypothesized that this may be due to direct effects of the FGFR3 mutation on cerebellar morphogenesis.
\end{abstract}

ABBREVIATIONS: PCF = posterior cranial fossa; PCFBV = posterior cranial fossa brain volume; STBV = supratentorial brain volume

A chondroplasia is the most common form of inherited disproportionate short stature affecting approximately 250,000 people worldwide. It is an autosomal dominant condition. The responsible mutation is almost always a glycine-toarginine substitution at codon 380 in the transmembrane domain of the fibroblast growth factor receptor 3 (FGFR3) gene, which results in overactivation of the receptor. Neuroimaging is frequently performed in children with achondroplasia because there are known associated intracranial abnormalities. $^{1-4}$

Macrocerebellum, an abnormally enlarged cerebellum, while rare, is associated with many syndromic conditions ${ }^{5-12}$ and has also been described as an isolated finding. ${ }^{13-15}$ It has, however, never been reported in achondroplasia. We suspected an increased cerebellar volume in patients with achondroplasia based on observed qualitative MR imaging findings. However,

Received August 14, 2019; accepted after revision November 2

From the Departments of Medical Imaging (H.M.P., A.M.F., S.K.) and Neurosurgery (J.Y.-M.Y.), The Royal Children's Hospital, Parkville, Victoria, Australia;

Neuroscience Research (J.Y.-M.Y.) and Developmental Imaging (J.Y.-M.Y., J.C.), Murdoch Children's Research Institute, Parkville, Victoria, Australia; Department of Paediatrics (J.Y.-M.Y.), University of Melbourne, Parkville, Victoria, Australia; Department of Perinatal Medicine (A.M.F.), Mercy Hospital for Women, Heidelberg, Victoria, Australia; and Department of Diagnostic Imaging (S.K.), Monash Health, Clayton, Victoria, Australia.

Dr Joseph Yuan-Mou Yang receives grant support from the Royal Children's Hospital Foundation (RCH1000)

Please address correspondence to Heather Michelle Pascoe, MD, Department of Medical Imaging, The Royal Children's Hospital, 50 Flemington Rd, Parkville,

Victoria, Australia, 3052; e-mail: pascoeh@gmail.com

http://dx.doi.org/10.3174/ajnr.A6369 given the high incidence of skull base deformities in achondroplasia, the possibility that these were contributing to the MR imaging appearances by causing crowding of the posterior cranial fossa (PCF) structures was considered. Skull base deformation occurs due to impaired endochondral ossification and premature fusion of the posterior fossa synchondroses. ${ }^{1,3} \mathrm{~A}$ recent quantitative study of the PCF in patients with achondroplasia, however, did not show a decreased bony PCF volume compared with controls but did confirm an increased cerebellar volume. $^{3}$

We hypothesized that the appearances described in macrocerebellum are present to varying degrees in patients with achondroplasia and that these are due to a true increase in the cerebellar volume rather than secondary to skull base changes. In our case series, we describe the qualitative imaging features suggestive of macrocerebellum and use volumetric analysis to confirm our findings.

\section{Case Series}

A retrospective MR imaging data base search was conducted using the Radiology Information System at the Medical Imaging Department, The Royal Children's Hospital, Melbourne, Australia. We identified all children (from birth to 18 years of age) who had a diagnosis of achondroplasia and an MR imaging study of the brain performed for any indication between January 2000 and November 2018. The study was approved by our institutional Human Research Ethics Committee as a clinical audit. 


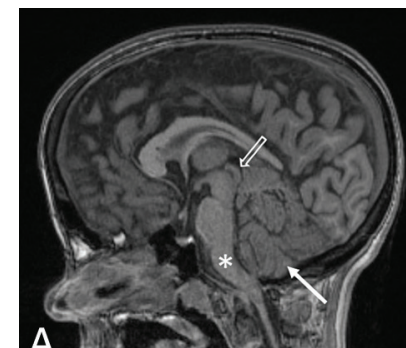

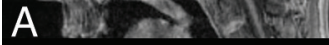

B
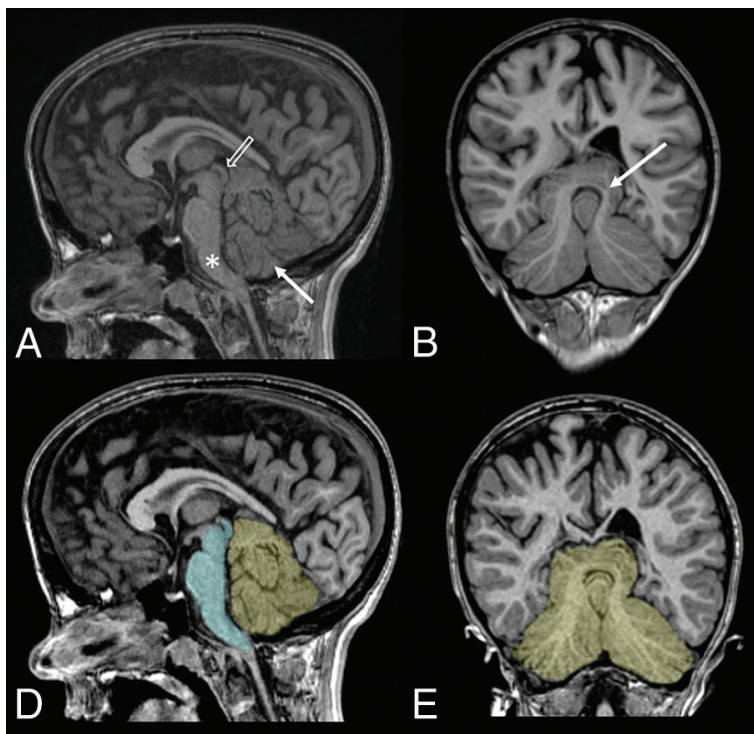

$\mathrm{C}$
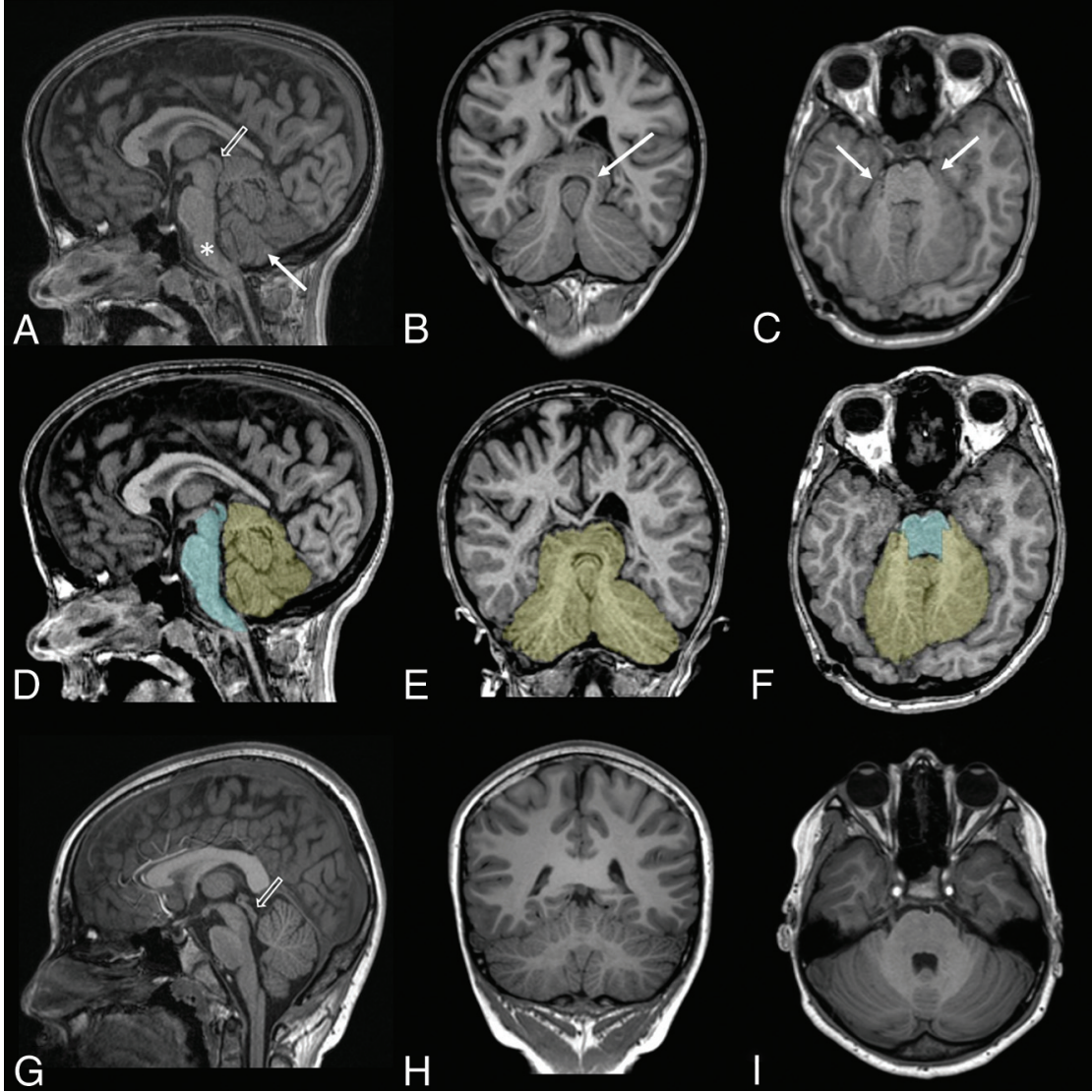

FIG 1. TIWIs of an 11-year-old child with achondroplasia $(A-C)$ and the same TTWIs with superimposed cerebellum (yellow) and brainstem (cyan) segmentations ( $D-F$ ). T7Wls from a healthy 11year-old child $(G-l)$ are provided for comparison. Extension of the cerebellar hemispheres inferior to the vermis is seen on the midsagittal plane (arrow in A); extension of the superomedial portions of the hemispheres above the tentorium cerebelli is seen on the coronal plane (arrow in B); and wrapping of the cerebellar hemispheres around the brainstem with extension around the lateral aspects of the brainstem at the level of the middle cerebellar peduncles is seen on the axial plane (arrows in C). Also seen on the midsagittal plane $(A)$ are effacement of the retroand infravermian subarachnoid CSF spaces, mass effect on the brainstem (which is anteriorly bowed [asterisk] with narrowing of the prepontine cistern and partial effacement of the fourth ventricle), and shortening of the midbrain with distortion of the tectal plate (open arrow). Note the normal morphology of the midbrain and tectal plate in $G$ (open arrow).

The inclusion criteria were a diagnosis of achondroplasia and the availability of a diagnostic-quality MR examination of the brain in the study period. If the patient had $>1 \mathrm{MR}$ imaging in the study period, the most recent MR imaging was used. Twentythree patients were included (12 boys and 11 girls). Ages ranged from 3 months to 15 years. An age- and sex-matched healthy control was selected for each patient. The controls were identified from the MR imaging database at The Royal Children's Hospital using the following criteria: 1) normal brain anatomy, and 2) absence of a neurologic disorder. Reasons for their MR imaging referral were either sensorineural hearing loss or headache. MR imaging studies were performed on a 1.5 or $3 \mathrm{~T}$ scanner.

Both qualitative and quantitative analyses were performed.

For qualitative analysis, the volumetric T1WIs were retrospectively evaluated by a pediatric radiology fellow (H.M.P.) and 2 pediatric neuroradiologists (S.K. and A.M.F.), and the results were consensus-based. The presence of the following qualitative imaging features (Fig $1 A-C$ ) was assessed:

1. Extension of the cerebellar hemispheres inferior to the vermis (on the midsagittal plane; Fig $1 A$ ).

2. Extension of the superomedial portions of the cerebellar hemispheres above the tentorium cerebelli (on the coronal plane; Fig $1 B$ ).

3. Wrapping of the cerebellar hemispheres around the brainstem with extension around the lateral aspects of the brainstem at the level of the middle cerebellar peduncles (on the axial plane; Fig $1 C$ ).

4. Effacement of the retro- and infravermian subarachnoid CSF spaces (on the midsagittal plane; Fig $1 A$ ).

5. Apparent shortening of the midbrain with or without distortion of the tectal plate (on the midsagittal plane; Fig 1A).

6. Mass effect on the brainstem (on the midsagittal plane; Fig $1 A$ ). The features assessed were anterior bowing of the brainstem, narrowing of the prepontine cistern, and effacement of the fourth ventricle.

Quantitative analysis of the cerebellar and brainstem volumes was performed in each patient and the ageand sex-matched healthy control using automated brain tissue segmentation. T2WIs were used for analyzing patients younger than 1 year of age, and T1WIs for patients older than 1 year of age. All T2WIs used for the younger subjects had $0.4-\mathrm{mm}$ in-plane resolution and $4-\mathrm{mm}$ slice thickness. All 3D T1WIs had similar $(0.5-0.9 \mathrm{~mm})$ in-plane resolution and 0.9-mm slice thickness. The imaging sequence used and the resolution were comparable between the patient and the paired control to avoid introducing bias in our quantitative analysis. Morphologically Adaptive Neonatal Tissue Segmentation (MANTIS) software (https://www.mantisoftware.com/) ${ }^{16}$ was used in the younger group, and a surface-based brain parcellation software, FreeSurfer Version 6.0 (http://surfer.nmr.mgh.harvard. edu), ${ }^{17}$ was used in the older group. Manual editing was subsequently performed to ensure anatomic accuracy. This was performed by the neurosurgery fellow (J.Y.-M.Y.) and reviewed by a pediatric radiology fellow (H.M.P.) and a pediatric neuroradiologist (S.K.). The cerebellar segmentation included both the cerebellar hemispheres and vermis. The brainstem segmentation included the midbrain, pons, and medulla. The posterior cranial fossa brain volume (PCFBV) was derived by summing the cerebellum and brainstem volumes. 


\begin{tabular}{lc}
\multicolumn{1}{c}{ Macrocerebellum Imaging Features on Different Imaging Planes } & $\begin{array}{c}\text { No. of Patients with Imaging Features } \\
\text { Present (\% Total) (Total }=23 \text { Patients) }\end{array}$ \\
\hline Extension of the cerebellar hemispheres inferior to the vermis (midsagittal) & $23(100.0)$ \\
Extension of the superomedial portions of the hemispheres above the & $16(69.6)$ \\
tentorium cerebelli (coronal) & $20(87.0)$ \\
Wrapping of the cerebellar hemispheres around the brainstem (axial) & $22(95.7)$ \\
Effacement of the retro- and infravermian subarachnoid CSF spaces (midsagittal) & $8(34.8)$ \\
Shortening of the midbrain (midsagittal) & $3(13.0)$ \\
Distortion of the tectal plate (midsagittal) & $7(30.4)$ \\
Mass effect on the brain stem (midsagittal) & \\
\hline
\end{tabular}

Table 2: Quantitative analysis of the cerebellum and brainstem

\begin{tabular}{|c|c|c|c|}
\hline Variables & $\begin{array}{c}\text { Patient } \\
\text { (Mean } \pm \text { SD) } \\
\end{array}$ & $\begin{array}{c}\text { Control } \\
\text { (Mean } \pm \text { SD) }\end{array}$ & $P$ Value $^{\mathrm{a}}$ \\
\hline Age (yr) & $5.38 \pm 4.39$ & $5.43 \pm 4.46$ & .43 \\
\hline Cerebellum (mL) & $137.85 \pm 29.55$ & $127.00 \pm 27.78$ & $.009^{\mathrm{a}}$ \\
\hline Brainstem (mL) & $19.28 \pm 3.96$ & $19.56 \pm 4.91$ & .92 \\
\hline PCFBV $(\mathrm{mL})$ & $157.14 \pm 33.30$ & $146.55 \pm 32.16$ & $.02^{\mathrm{a}}$ \\
\hline STBV (mL) & $1097.56 \pm 171.54$ & $1001.05 \pm 187.58$ & $<.001^{\mathrm{a}}$ \\
\hline
\end{tabular}

Note:-PCFBV indicates posterior cranial fossa brain volume; STBV, supratentorial brain volume; SD, standard deviation.

${ }^{\text {a }}$ Statistical significance $(P<.05)$.

The supratentorial brain volume (STBV) was also calculated for all cases. This was performed using the brain tissue classification tool from SPM12 (http://www.fil.ion.ucl.ac.uk/spm/software/ spm 12$)^{18}$ to calculate the total brain volume, and the PCFBV was then subtracted from this.

Statistical analysis was performed using the R statistical and computing software and graphics (http://www.r-project.org). Group summary statistics were expressed in means and SDs. Comparison between the patients and their age- and sex-matched controls was analyzed using the paired Wilcoxon rank sum test. $P$ values $<.05$ were considered statistically significant.

Results of the qualitative neuroimaging features of macrocerebellum are summarized in Table 1 . Each of the MR imaging findings was recorded as present or not. Figure $1 A-C$ demonstrates the qualitative MR imaging findings, with a healthy control for comparison shown in Fig 1G-I.

The results of quantitative analysis are summarized in Table 2. Figure $1 D-F$ demonstrates the cerebellar and brainstem segmentations in standard orthogonal 2D MR imaging planes. Figure 2 demonstrates the cerebellar and brainstem segmentation as a $3 \mathrm{D}$ volume rendering.

The achondroplasia patients had a significantly larger PCFBV compared with their age- and sex-matched healthy controls $(P=.02)$. This volume difference was due to a larger cerebellum $(P=.009)$. There was no brainstem volume difference between the 2 study groups $(P=.92)$. The patients with achondroplasia also had significantly larger STBVs compared with their age- and sex-matched healthy controls $(P<.001)$.

\section{DISCUSSION}

The prevalence of skull base changes in patients with achondroplasia is well-documented; however, the morphology of the PCF content has not been widely reported. ${ }^{3}$ In particular, appearances described in macrocerebellum, ${ }^{19}$ such as those we observed, have not been described in this patient group.
The cerebellar enlargement in macrocerebellum has been reported to mainly involve the cerebellar hemispheres ${ }^{13,15}$ and has been described as an isolated finding ${ }^{13-15}$ as well as in many syndromic conditions. ${ }^{5-12}$ As a consequence of the cerebellar hemispheric enlargement, the hemispheres extend into available surrounding spaces, resulting in wrapping around the brainstem as well as upward and/or downward herniation. ${ }^{15}$ All of the patients with achondroplasia in our study demonstrated some of these qualitative signs of apparent cerebellar hemisphere enlargement. Ancillary changes of apparent macrocerebellum observed in the PCF were the following: effacement of the retro- and infravermian subarachnoid CSF spaces; apparent shortening of the midbrain, with associated distortion of the tectal plate in severe cases; and mass effect on the brainstem. Most of these features have been previously described in macrocerebellum. ${ }^{5,19}$

On the basis of our qualitative results, it remained possible that deformation of the bony PCF causing overcrowding could be responsible for the MR imaging appearance of an apparent macrocerebellum. However, in line with the findings by Calandrelli et $\mathrm{al}^{3}{ }^{3}$ we confirmed that compared with healthy controls, cerebellar volumes were increased in patients with achondroplasia, indicating true macrocerebellum. In contradistinction to Calandrelli et al, however, we found no difference in the brainstem volume between patients and controls. This may be due to the small number of cases in both series; thus, larger series would be needed to establish whether there is any brainstem involvement in achondroplasia.

Fibroblast growth factor receptors are transmembrane tyrosine kinases that are expressed in the developing brain. ${ }^{20}$ They are encoded by 4 genes (FGFR1-4), ${ }^{21}$ including the FGFR3 gene, which is mutated in achondroplasia. Fibroblast growth factors bind to the fibroblast growth factor receptors, and this signaling is an important regulator of cerebral cortex development. ${ }^{22}$ Studies have demonstrated malformations involving the cerebral cortex, such as expansion of the occipitotemporal cortex $^{22}$ and oversulcation of the mesiotemporal lobes ${ }^{4}$ in the presence of activating FGFR3 mutations. These findings may also explain our finding of significantly higher suptratentorial brain volume in patients with achondroplasia compared with healthy controls.

Less well-known is that fibroblast growth factor signaling has also been shown in mice to have an important role in regulating the formation of the cerebellum from the early embryonic period into early postnatal life. ${ }^{23}$ Three neural layers are present in the adult cerebellar cortex (the molecular, Purkinje cell, and granular 


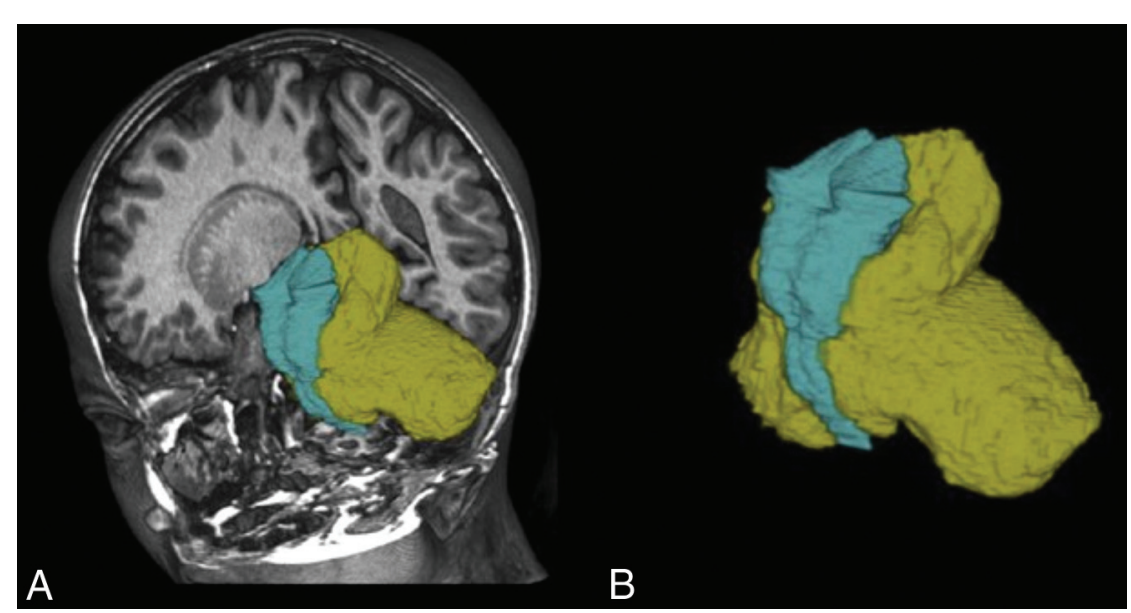

FIG 2. Cerebellum (yellow) and brainstem (cyan) segmentations of the same patient with achondroplasia as in Fig 1, shown as 3D volume renders.

and supratentorial features. Further pathologic and genetic studies will be required to gain a better understanding of our observations.

\section{ACKNOWLEDGMENTS}

We thank the support from The Royal Children's Hospital Foundation, Murdoch Children's Research Institute, The University of Melbourne Department of Pediatrics, and the Victorian Government's Operational Infrastructure Support Program.

\section{REFERENCES}

1. Kao SC, Waziri MH, Smith WL, et al. MR imaging of the craniovertebral junction, cranium, and brain in chil-

cells layers), and most of the cerebellar neurons migrate to the cortex from the ventricular zone during the early embryonic period under the influences of molecular signaling, including fibroblast growth factor signaling. In the mouse cerebellum, FGFR3 has been detected in the cerebellum as early as embryonic day 12.5. FGFR3 is then expressed at varying levels in different parts of the cerebellar nuclei and cortical layers throughout the remainder of the prenatal period extending to 21 days postnatally. ${ }^{23}$ Shazeeb et $\mathrm{al}^{24}$ reported no FGFR3 expression in the cerebellum of 3-week-old mice.

There is no current literature on the appearance of the cerebellum in achondroplasia. However, given our qualitative and quantitative findings, the known expression of FGFR3 in the cerebellum during early development in mice, and the known growth-promoting effects of FGFR3 mutations in the supratentorial compartment, we hypothesized that macrocerebellum in achondroplasia may be due to the direct effects of the FGFR3 mutation on cerebellar morphogenesis. Further genetic and pathologic studies are required to substantiate or refute this hypothesis.

A major limitation of our study is that it is retrospective, with a relatively small sample size. The prevalence of macrocerebellum in patients with achondroplasia can thus not be established from this study. Additionally, segmenting the cerebellar hemispheres and vermis separately was beyond the scope of the methodology of this study; thus, the relative contribution of each to the enlarged cerebellum cannot be determined.

\section{CONCLUSIONS}

Our study is the first to describe macrocerebellum with its typical appearance in children with achondroplasia. We confirmed an increase in the cerebellar volume quantitatively through volumetric analysis and hypothesized that macrocerebellum may be due to the direct effects of the FGFR3 mutation. Thus, macrocerebellum can be inferred when the qualitative imaging appearances we described are identified. These features should be sought when imaging patients with achondroplasia, alongside the other previously reported PCF dren with achondroplasia. AJR Am J Roentgenol 1989;153:565-69 CrossRef Medline

2. Pauli RM. Achondroplasia: a comprehensive clinical review. Orphanet J Rare Dis 2019;14:1 CrossRef Medline

3. Calandrelli R, Panfili M, D'Apolito G, et al. Quantitative approach to the posterior cranial fossa and craniocervical junction in asymptomatic children with achondroplasia. Neuroradiology 2017;59:1031-41 CrossRef Medline

4. Manikkam SA, Chetcuti K, Howell KB, et al. Temporal lobe malformations in achondroplasia: expanding the brain imaging phenotype associated with FGFR3-related skeletal dysplasias. AJNR Am J Neuroradiol 2018;39:380-84 CrossRef Medline

5. Alqahtani E, Huisman TA, Boltshauser E, et al. Mucopolysaccharidoses type I and II: new neuroimaging findings in the cerebellum. Eur $J$ Paediatr Neurol 2014;18:211-17 CrossRef Medline

6. Poretti A, Snow J, Summers AC, et al. Joubert syndrome: neuroimaging findings in 110 patients in correlation with cognitive function and genetic cause. J Med Genet 2017;54:521-29 CrossRef Medline

7. Shiohama T, Fujii K, Miyashita T, et al. Brain morphology in children with nevoid basal cell carcinoma syndrome. Am J Med Genet A 2017;173:946-52 CrossRef Medline

8. Schaefer GB, Bodensteiner JB, Buehler BA, et al. The neuroimaging findings in Sotos syndrome. Am J Med Genet 1997;68:462-65 CrossRef Medline

9. Gripp KW, Hopkins E, Doyle D, et al. High incidence of progressive postnatal cerebellar enlargement in Costello syndrome: brain overgrowth associated with HRAS mutations as the likely cause of structural brain and spinal cord abnormalities. Am J Med Genet A 2010;152:1161-68 CrossRef Medline

10. Jones W, Hesselink J, Courchesne E, et al. Cerebellar abnormalities in infants and toddlers with Williams syndrome. Dev Med Child Neurol 2002;44:688-94 CrossRef Medline

11. van der Knaap MS, Salomons GS, Li R, et al. Unusual variants of Alexander's disease. Ann Neurol 2005;57:327-38 CrossRef Medline

12. Kau T, Karlo C, Güngör T, et al. Increased cerebellar volume in the early stage of fucosidosis: a case control study. Neuroradiology 2011;53:509-16 CrossRef Medline

13. Bodensteiner JB, Schaefer GB, Keller GM, et al. Macrocerebellum: neuroimaging and clinical features of a newly recognized condition. J Child Neurol 1997;12:365-68 CrossRef Medline

14. D’Arco F, Ugga L, Caranci F, et al. Isolated macrocerebellum: description of six cases and literature review. Quant Imaging Med Surg 2016;6:496-503 CrossRef Medline 
15. Izbudak I, Acer N, Poretti A, et al. Macrocerebellum: volumetric and diffusion tensor imaging analysis. Turk Neurosurg 2015;25:948-53 Medline

16. Beare RJ, Chen J, Kelly CE, et al. Neonatal brain tissue classification with morphological adaptation and unified segmentation. Front Neuroinform 2016;10:12 CrossRef Medline

17. Fischl B. FreeSurfer. Neuroimage 2012;62:774-81 CrossRef Medline

18. Ashburner J, Friston KJ. Unified segmentation. Neuroimage 2005; 26:839-51 CrossRef Medline

19. Poretti A, Mall V, Smitka M, et al. Macrocerebellum: significance and pathogenic considerations. Cerebellum 2012;11:1026-36 CrossRef Medline

20. Hevner RF. The cerebral cortex malformation in thanatophoric dysplasia: neuropathology and pathogenesis. Acta Neuropathol 2005;110:208-21 CrossRef Medline
21. Zhang X, Ibrahimi OA, Olsen SK, et al. Receptor specificity of the fibroblast growth factor family: the complete mammalian FGF family. J Biol Chem 2006;281:15694-700 CrossRef Medline

22. Thomson RE, Kind PC, Graham NA, et al. FGF receptor 3 activation promotes selective growth and expansion of occipitotemporal cortex. Neural Dev 2009;4:4 CrossRef Medline

23. Yaguchi Y, Yu T, Ahmed MU, et al. Fibroblast growth factor (FGF) gene expression in the developing cerebellum suggests multiple roles for FGF signaling during cerebellar morphogenesis and development. Dev Dyn 2009;238:2058-72 CrossRef Medline

24. Shazeeb MS, Cox MK, Gupta A, et al. Skeletal characterization of the FGFR3 mouse model of achondroplasia using micro-CT and MRI volumetric imaging. Sci Rep 2018;8:469 CrossRef Medline 